\title{
Prevalence of Epilepsy in the Elderly: The Rotterdam Study
}

\author{
*Annette de la Court, *†Monique M. B. Breteler, $\ddagger$ Harry Meinardi, §W. Allen Hauser, \\ and $* \dagger$ Albert Hofman \\ *The Netherlands Institute for Health Sciences, Erasmus University Medical School, Rotterdam; †Department of \\ Epidemiology \& Biostatistics, Erasmus University Medical School, Rotterdam; $¥$ Department of Epileptology, \\ Catholic University Nȳmegen, Nȳmegen, The Netherlands; and §G.H. Sergievsky Center, Columbia University, \\ New York, New York, U.S.A.
}

Summary: We assessed the prevalence of epilepsy in an elderly population in The Netherlands. The study was conducted from 1991 to 1993 as part of the Rotterdam Study, a population-based door-to-door study of all elderly people living in Ommoord, a suburb of Rotterdam, and included 5,559 persons aged 55-95 years. All subjects were screened for epilepsy through direct questions regarding the existence of epilepsy and antiepileptic drug (AED) use, in addition to relevant questions from the World Health Organization (WHO) protocol for epidemiologic studies of neurologic diseases. Further evaluation of screen positives was made by a panel of 1 study physician and 4 epileptologists, who also classified all confirmed cases of epilepsy according to the classifications of the International League Against Epilepsy (ILAE). The overall prevalence of active epilepsy in our study population was $0.9 \%$ including special syndromes and $0.8 \%$ excluding special syndromes. The prevalence increased with age from $0.7 \%$ for those aged 55-64 years to $1.2 \%$ for those aged 85-94 years. The increase with age was detected among men and women both. Our study confirms other findings showing that the prevalence of active epilepsy increases with age in the elderly. The prevalence figures in our study were high as compared with those of other population-based studies. Epilepsy appears to be a major cause of morbidity in the elderly. Key Words: Epilepsy-Population studies-Prevalence-ScreeningSeizures.
The prevalence of epilepsy in the elderly has been investigated in only a few studies. Hauser and Kurland $(1,2)$ and Tallis et al. (3) reported that the prevalence and incidence of epilepsy increase with age, but this was not confirmed by other investigators $(4,5)$. In a large primary-care-based study of people aged $\geqslant 60$ years in the United Kingdom, Tallis et al. reported an increase in age-adjusted prevalence and incidence figures of epilepsy, making it the third most common neurological disorder (after stroke and dementia) in this age group (3). We wished to assess the prevalence of epilepsy in an elderly Dutch population.

\section{METHODS}

\section{Study population}

The investigation was conducted as part of the ongoing Rotterdam Study, a prospective follow-up

Received May 18, 1995; revision accepted September 11, 1995

Address correspondence and reprint requests to Dr. M. M. B. Breteler at Department of Epidemiology and Biostatistics, Erasmus University Medical School, P.O. Box 1738, 3000 DR Rotterdam, The Netherlands. survey of the total population aged $\geqslant 55$ years in the district of Ommoord in Rotterdam, the Netherlands (6). The baseline survey took place from June 1990 until June 1993. Informed consent and permission to obtain relevant information from treating physicians were obtained from all participants. Throughout the Rotterdam Study, subjects were surveyed in random clusters. Epilepsy screening was incorporated at the time of the 25th random survey in June 1991. The total eligible population consisted of 7,129 persons. Because the number of subjects aged $\geqslant 95$ years was to small to determine an accurate prevalence figure, we excluded this age group from our analysis. Therefore our total eligible population consisted of 7,081 persons, 5,559 (79\%) of whom participated in the epilepsy screening.

\section{Screening for epilepsy}

The standard Rotterdam Study protocol consisted of a home interview followed by physical examinations in a research center. The screening for epilepsy was conducted during the home interview by a trained research assistant and consisted of one 
question directly inquiring about epilepsy (Have you ever experienced epilepsy or had an epileptic fit?) and three questions regarding possible symptoms of epilepsy that were taken from the World Health Organization (WHO) research protocol for the screening of neurological disorders in developing countries (7), as follows: Have you ever lost consciousness? Have you ever had episodes where you lost contact with your surroundings? and Have you ever had any shaking of your arms and legs which you could not control? In addition, all current drug prescriptions were registered and classified according to the Anatomical Therapeutic Chemical (ATC) classification index (8). We defined as screen-positive for epilepsy all persons who (a) self reported epilepsy or fits, or (b) used antiepileptic drugs (AEDs: ATC-code N03), or (c) answered yes to at least two of the three WHO questions.

\section{Diagnostic workup and classification of epilepsy}

For persons who reported having had epilepsy or seizures during their life $(n=97)$, as well as for persons receiving AEDs without reporting epilepsy ( $n=24)$, additional information was obtained from general practitioner's and specialists' medical records, including EEG and computed tomography (CT) scan reports. For 22 subjects, no further information was available from medical records and they were contacted by telephone by a specially trained physician who took a detailed medical and seizure history. A similar procedure was used for subjects who gave positive responses to at least two of the WHO screening questions but who neither reported having epilepsy or receiving AEDs $(n=192)$. Persons without a telephone, those who did not answer the telephone on several occasions, and those who were unable to give a reliable history by telephone were invited to an in-person interview, either in their home or at the research center. If the history suggested the possibility of epilepsy, the subject was reinterviewed by 1 of 4 epileptologists (Drs. D. J. Beintema, C. A. E. H. van Oorschot, A. van Wieringen, and J. M. de Wilde-Ockeloen), and additional information was obtained from the general practitioner and other medical records.

All 313 screen-positive persons were subsequently reviewed by a panel consisting of the study physician and the 4 epileptologists. From all available information, the panel then decided whether a subject had epilepsy and, if so, classified the epilepsy according to the classification of the International League Against Epilepsy (ILAE) (9). Single seizures, treated or not, were classified as "epilepsy-special syndromes" according to 1989 ILAE guidelines (9). Active epilepsy was defined as having had a seizure within 5 years of prevalence date, regardless of treatment (10).

\section{Data analysis}

Prevalence was calculated by gender and 10-year age groups. We calculated the prevalence of active epilepsy and of lifetime epilepsy, which includes both active epilepsy and epilepsy in remission. Prevalence of active epilepsy was calculated both including and excluding special syndromes. To compare our results with those of other published studies, we also calculated the age-specific prevalences of active epilepsy, defined as having had a seizure within 5 years of prevalence date or current use of AEDs. The proportion of each ILAE subtype of epilepsy was reported by gender.

\section{RESULTS}

Table 1 shows the age and gender distribution of the study population and the number of screenpositive individuals. Of the total population of 5,559 persons screened, 313 persons $(5.6 \%)$ were screen positive. No screen-positive men were in the highest age category. Overall, $97(1.7 \%)$ persons reported epilepsy, $24(0.4 \%)$ received AEDs without

TABLE 1. Age distribution and screening results of the study population

\begin{tabular}{|c|c|c|c|c|c|}
\hline \multirow[b]{2}{*}{ Parameter } & \multicolumn{5}{|c|}{ Age groups (yr) } \\
\hline & $55-64$ & $65-74$ & $76-84$ & $85-94$ & Total \\
\hline No. of subjects & 2,125 & 1,916 & 1,105 & 413 & 5,559 \\
\hline Proportion of women $(\%)$ & 56 & 55 & 65 & 80 & 59 \\
\hline No. with self-reported epilepsy (\%) & $38(1.7)$ & $37(1.9)$ & $14(1.3)$ & $8(1.9)$ & $97(1.7)$ \\
\hline No. receiving AEDs $(\%)^{a}$ & $19(0.9)$ & $23(1.2)$ & $13(1.2)$ & $6(1.5)$ & $61(1.1)$ \\
\hline No. of WHO screen positives $(\%)^{b}$ & $97(4.6)$ & $80(4.2)$ & $45(4.1)$ & $15(3.6)$ & $237(4.3)$ \\
\hline No. of screen positives $(\%)^{c}$ & $121(5.7)$ & $115(6.0)$ & $56(5.1)$ & $21(5.1)$ & $313(5.6)$ \\
\hline
\end{tabular}

AED, antiepileptic drug; WHO, World Health Organization.

${ }^{a}$ Regardless of self-reported epilepsy.

${ }^{b}$ Regardless of self-reported epilepsy and AED use: defined as having answered positively to at least two of the three WHO screening questions on epilepsy.

${ }^{c}$ Defined as self-reported epilepsy or AED use or WHO screen positive. 
TABLE 2. Results of verification for all screen-positive subjects

\begin{tabular}{lcccr}
\hline & \multicolumn{4}{c}{ Final diagnosis } \\
\cline { 2 - 5 } $\begin{array}{c}\text { Screen-positive } \\
\text { categories }\end{array}$ & Epilepsy & $\begin{array}{c}\text { No } \\
\text { epilepsy }\end{array}$ & Missing & Total \\
\hline $\begin{array}{l}\text { Self-reported epilepsy } \\
\begin{array}{c}\text { AED use without } \\
\text { self-reported }\end{array}\end{array}$ & 70 & 20 & 7 & 97 \\
$\begin{array}{l}\text { epilepsy } \\
\text { WHO screen positive }\end{array}$ & 4 & 20 & 0 & 24 \\
$\begin{array}{l}\text { without } \\
\text { self-reported }\end{array}$ & & & & \\
$\begin{array}{l}\text { epilepsy or AED } \\
\text { use }\end{array}$ & 11 & 180 & 1 & 192 \\
Total & 85 & 220 & 8 & 313 \\
\hline
\end{tabular}

Abbreviations as in Table 1.

${ }^{a}$ Defined as having answered positively to at least two of the three WHO screening questions on epilepsy.

reporting epilepsy, and $192(3.5 \%)$ persons were screen positive because of positive answers to the WHO questions even though they did not report epilepsy or treatment with AEDs.

Table 2 shows results after verification of all screen-positive subjects. We confirmed 85 cases of epilepsy, or $27 \%$ of all screen positives. For 27 persons $(28 \%)$ who reported epilepsy, the diagnosis could not be confirmed. Eleven cases showed clear evidence from neurologists' records for a nonepileptic cause of symptoms: sleep apnea, transient ischemic attack $(n=2)$, depression, psychogenic attacks $(n=2)$, muscle headaches, narcolepsy, amaurosis fugax with migraine, nocturnal urinary incontinence, and syncope. For the remaining 16 persons, no information regarding possible epilepsy or other paroxysmal disorder could be detected. Nine of them were reinterviewed by 1 of the epileptologists, who made a specific nonepilepsy diagno-
TABLE 4. Summary of special syndromes in the Rotterdam Study

\begin{tabular}{|c|c|}
\hline $\begin{array}{c}\text { Etiology } \\
\text { (no. of subjects) }\end{array}$ & Associated condition \\
\hline Acute symptomatic (7) & $\begin{array}{l}\text { Eclampsia, acute CVA, fever, } \\
\text { influenza encephalitis, alcohol } \\
\text { withdrawal (2), cerebral anoxia }\end{array}$ \\
\hline Remote symptomatic (3) & $\begin{array}{l}\text { CVA }>1 \text { year before seizure, } \\
\text { Parkinson's disease, subdural } \\
\text { hematoma }\end{array}$ \\
\hline Cryptogenic (10) & $\begin{array}{l}\text { Abnormal EEG or CT scan; no } \\
\text { known antecedent cause (5); } \\
\text { no abnormality on EEG or CT } \\
\text { scan; no known antecendent } \\
\text { cause (5) }\end{array}$ \\
\hline
\end{tabular}

Total (20)

CVA, cerebrovascular accident; CT, computed tomography.

sis. No further information could be obtained in 7 individuals. Of the 24 subjects who received AEDs without reporting epilepsy, we concluded that only 4 had epilepsy. The reasons for treatment with AEDs for conditions other than epilepsy were diverse: 3 persons were prescribed clonazepam for restless legs syndrome, 7 persons received carbamazepine (CBZ) for trigeminal or hypoglossal neuralgia, 1 subject received $\mathrm{CBZ}$ for manic-depressive psychosis; 1 was prescribed valproate (VPA) prophylactically after herpes encephalitis without clinical or EEG evidence of epilepsy, 5 persons with mental disorders were long-term barbiturate users, 2 persons were prescribed $\mathrm{CBZ}$ for polyneuropathy, and 1 subject could not be verified as an AED user.

Table 3 summarizes the types of epilepsies for both active and lifetime epilepsy and for men and women. Localization-related epilepsy was the most

TABLE 3. Subclassification of epilepsies according to the ILAE

\begin{tabular}{|c|c|c|c|c|}
\hline \multirow[b]{2}{*}{ Epilepsy type according to ILAE classification } & \multicolumn{2}{|c|}{$\begin{array}{l}\text { Active epilepsy, } \\
\text { no. of subjects } \\
(\%)\end{array}$} & \multicolumn{2}{|c|}{$\begin{array}{l}\text { Lifetime epilepsy, } \\
\text { no. of subjects } \\
(\%)\end{array}$} \\
\hline & Men & Women & Men & Women \\
\hline I Localization-related epilepsies & $17(73.9)$ & $18(64.3)$ & $19(55.9)$ & $21(41.2)$ \\
\hline I-1 Idiopathic & 0 & 0 & 0 & 0 \\
\hline I-2 Symptomatic & 10 & 8 & 11 & 10 \\
\hline I-3 Cryptogenic & 7 & 10 & 8 & 11 \\
\hline II Generalized epilepsies & $1(4.4)$ & $2(7.1)$ & $3(8.8)$ & $2(3.9)$ \\
\hline II-1 Idiopathic & 0 & 1 & 1 & 1 \\
\hline II-2 Cryptogenic or symptomatic & 0 & 0 & 0 & 0 \\
\hline II-3 Symptomatic & 1 & 1 & 2 & 1 \\
\hline III Epilepsies undetermined whether focal or generalized & $0(0.0)$ & $4(14.3)$ & $4(11.8)$ & $16(31.4)$ \\
\hline III-1 With both focal and generalized seizures & 0 & 0 & 0 & 0 \\
\hline III-2 Undetermined seizure type & 0 & 4 & 4 & 16 \\
\hline IV Special syndromes & $5(21.7)$ & $4(14.3)$ & $8(23.5)$ & $12(23.5)$ \\
\hline IV-1 Single seizure, not followed by treatment with AEDs & 1 & 2 & 3 & 6 \\
\hline IV-2 Single seizure, followed by treatment with AEDs & 4 & 2 & 5 & 6 \\
\hline Total & $23(100 \%)$ & $28(100 \%)$ & $34(100 \%)$ & $51(100 \%)$ \\
\hline
\end{tabular}

ILAE, International League Against Epilepsy; AEDs, antiepileptic drugs. 
TABLE 5. Prevalence of lifetime and active epilepsy by gender and age [cases per 1,000 (no. of cases)]

\begin{tabular}{|c|c|c|c|c|c|c|c|c|}
\hline \multirow{3}{*}{$\begin{array}{l}\text { 10-Year } \\
\text { age group }\end{array}$} & \multicolumn{5}{|c|}{ Men } & \multicolumn{3}{|c|}{ Women } \\
\hline & \multirow{2}{*}{$\begin{array}{l}\text { No. of } \\
\text { subjects }\end{array}$} & \multicolumn{2}{|c|}{$\begin{array}{l}\text { Including special } \\
\text { syndromes }\end{array}$} & \multicolumn{2}{|c|}{$\begin{array}{l}\text { Excluding special } \\
\text { syndromes }\end{array}$} & \multirow{2}{*}{$\begin{array}{l}\text { No. of } \\
\text { subjects }\end{array}$} & \multicolumn{2}{|c|}{$\begin{array}{l}\text { Including special } \\
\text { syndromes }\end{array}$} \\
\hline & & Lifetime & Active & Lifetime & Active & & Lifetime & Active \\
\hline $55-64$ & 932 & $13.9(13)$ & \multirow{4}{*}{$\begin{array}{c}6.4(6) \\
11.8(11) \\
15.4(6) \\
0\end{array}$} & $9.7(9)$ & $4.3(4)$ & 1,193 & $16.8(20)$ & $7.5(9)$ \\
\hline $65-74$ & 855 & $16.4(14)$ & & $12.9(11)$ & $10.5(9)$ & 1,061 & $17.0(18)$ & $7.5(8)$ \\
\hline $75-84$ & 390 & $17.9(7)$ & & $15.4(6)$ & $12.8(5)$ & 715 & $9.8(7)$ & $8.4(6)$ \\
\hline $85-94$ & 83 & 0 & & 0 & 0 & 330 & $18.2(6)$ & $15.2(5)$ \\
\hline \multirow[t]{2}{*}{ Total } & 2,260 & $15.0(34)$ & $10.2(23)$ & $11.5(26)$ & $8.0(18)$ & 3,299 & $15.5(51)$ & $8.5(28)$ \\
\hline & \multicolumn{2}{|c|}{ Women } & \multicolumn{6}{|c|}{ All } \\
\hline \multirow{2}{*}{$\begin{array}{l}\text { 10-Year } \\
\text { age group }\end{array}$} & \multicolumn{2}{|c|}{$\begin{array}{l}\text { Excluding special } \\
\text { syndromes }\end{array}$} & \multirow{2}{*}{\multicolumn{2}{|c|}{$\begin{array}{l}\text { No. of } \\
\text { subjects }\end{array}$}} & \multicolumn{2}{|c|}{$\begin{array}{l}\text { Including special } \\
\text { syndromes }\end{array}$} & \multicolumn{2}{|c|}{$\begin{array}{l}\text { Excluding special } \\
\text { syndromes }\end{array}$} \\
\hline & Lifetime & Active & & & Lifetime & Active & Lifetime & Active \\
\hline $55-64$ & $12.6(15)$ & $7.5(9)$ & & & $16.0(33)$ & $7.1(15)$ & $11.3(24)$ & $6.1(13)$ \\
\hline $65-74$ & 12.3 (13) & $6.6(7)$ & & & $16.2(32)$ & 9.9 (19) & $12.5(24)$ & $8.4(16)$ \\
\hline $75-84$ & $8.4(6)$ & $7.0(5)$ & & & $12.7(14)$ & $10.9(12)$ & $10.9(12)$ & $9.0(10)$ \\
\hline $85-94$ & $15.2(5)$ & $12.1(4)$ & & & $14.5(6)$ & $12.1(5)$ & $12.1(5)$ & $9.7(4)$ \\
\hline Total & $11.8(39)$ & $7.6(25)$ & & & $15.3(85)$ & $9.2(51)$ & $11.7(65)$ & $7.7(43)$ \\
\hline
\end{tabular}

${ }^{a}$ Defined as at least one seizure within 5 years of prevalence date, regardless of treatment.

common type in both men and women. The second most common type of active epilepsy in men was special syndrome, whereas in women it was both undetermined epilepsy and special syndrome. Generalized epilepsy was relatively uncommon in both sexes.

Table 4 summarizes all special syndromes. Of the 20 cases detected, 7 were acute symptomatic seizures, 3 of which were subsequently treated with AEDs [stroke, alcohol withdrawal $(n=2)]$. Three patients with remote symptomatic epilepsy were detected, all of whom received AEDs. The remaining 10 patients had no determinable antecedent cause, but 5 of them had abnormalities on EEG or CT scan; 1 of the 5 patients subsequently was started on AED treatment. Four of the 5 remaining subjects with no abnormality on EEG or CT scan were treated with AEDs.

The prevalence figures of epilepsy are shown in Table 5. We noted an overall lifetime prevalence of epilepsy in this 55-95-year-old population of 15.3 cases in 1,000 and an overall prevalence of active epilepsy of 9.2 cases in 1,000 . The prevalence of active epilepsy increased with advancing age, but the prevalence of lifetime epilepsy leveled off or even decreased in the highest age groups. When we excluded special syndromes, the overall prevalence for active epilepsy was 7.7 cases per 1,000 persons. When we included subjects treated with AEDs in the definition of active epilepsy, regardless of whether they had had a seizure in the last 5 years, the prevalence figures increased slightly (Table 6).

\section{DISCUSSION}

We screened a large elderly population and determined the prevalence of active and lifetime epilepsy in the group. The prevalence figures for active epilepsy were among the highest reported in the literature among elderly persons. Only Hauser et al. reported a higher rate for active epilepsy in persons aged $>75$ years $(2)$.

TABLE 6. Prevalence of active epilepsy by gender and age (cases per 1,000 persons)

\begin{tabular}{|c|c|c|c|c|c|c|c|c|c|}
\hline \multirow[b]{2}{*}{$\begin{array}{c}\text { 10-Year } \\
\text { age group }\end{array}$} & \multicolumn{3}{|c|}{ Men } & \multicolumn{3}{|c|}{ Women } & \multicolumn{3}{|c|}{ All } \\
\hline & $\begin{array}{l}\text { No. of } \\
\text { subjects }\end{array}$ & $\begin{array}{l}\text { Including } \\
\text { special } \\
\text { syndromes }\end{array}$ & $\begin{array}{l}\text { Excluding } \\
\text { special } \\
\text { syndromes }\end{array}$ & $\begin{array}{l}\text { No. of } \\
\text { subjects }\end{array}$ & $\begin{array}{l}\text { Including } \\
\text { special } \\
\text { syndromes }\end{array}$ & $\begin{array}{l}\text { Excluding } \\
\text { special } \\
\text { syndromes }\end{array}$ & $\begin{array}{l}\text { No. of } \\
\text { subjects }\end{array}$ & $\begin{array}{l}\text { Including } \\
\text { special } \\
\text { syndromes }\end{array}$ & $\begin{array}{l}\text { Excluding } \\
\text { special } \\
\text { syndromes }\end{array}$ \\
\hline $55-64$ & 932 & 8.6 & 6.4 & 1,193 & 9.2 & 8.4 & 2,125 & 8.9 & 7.5 \\
\hline $65-74$ & 855 & 14.0 & 11.7 & 1,061 & 9.4 & 8.5 & 1,916 & 11.5 & 9.9 \\
\hline $75-84$ & 390 & 15.4 & 12.8 & 715 & 8.4 & 7.0 & 1,105 & 10.9 & 9.0 \\
\hline $85-94$ & 83 & 0.0 & 0.0 & 330 & 18.2 & 15.2 & 413 & 14.5 & 12.1 \\
\hline Total & 2,260 & 11.5 & 9.3 & 3,299 & 10.0 & 8.8 & 5,559 & 10.6 & 9.0 \\
\hline
\end{tabular}

Active epilepsy defined as at least one seizure within 5 years of prevalence date or antiepileptic drug use. 
TABLE 7. Prevalence of epilepsy in elderly subjects: A comparison of recent studies

\begin{tabular}{|c|c|c|c|c|c|c|c|}
\hline \multirow{2}{*}{$\begin{array}{c}\text { Country } \\
\text { (reference) }\end{array}$} & \multirow[b]{2}{*}{ Year } & \multirow[b]{2}{*}{ Study design } & \multirow{2}{*}{$\begin{array}{l}\text { Study size } \\
\text { (age, yr) }\end{array}$} & \multirow{2}{*}{$\begin{array}{c}\text { Age } \\
\text { group } \\
(\mathrm{yr})\end{array}$} & \multicolumn{2}{|c|}{$\begin{array}{c}\text { Prevalence } \\
\text { (cases per } 1,000 \text { ) }\end{array}$} & \multirow{2}{*}{$\begin{array}{l}\text { Criteria used to define epilepsy } \\
\text { and activeness of epilepsy }\end{array}$} \\
\hline & & & & & Active & Lifetime & \\
\hline $\begin{array}{l}\text { Rotterdam, The } \\
\text { Netherlands }\end{array}$ & 1994 & $\begin{array}{l}\text { Population based; WHO } \\
\text { screening protocol }\end{array}$ & $\begin{array}{l}N=5,559 \\
.(55+)\end{array}$ & $\begin{array}{l}55-64 \\
65-74 \\
75-84 \\
85-94\end{array}$ & $\begin{array}{rr}6.1 & 7.5 \\
8.4 & 9.9 \\
9.0 & 9.0 \\
9.7 & 12.1\end{array}$ & $\begin{array}{l}11.3 \\
12.5 \\
10.9 \\
12.1\end{array}$ & $\begin{array}{l}\text { Recurrent seizures, provoked and } \\
\text { unprovoked; active defined as } \\
\text { having had at least one seizure } \\
\text { in the past } 5 \text { years regardless of } \\
\text { treatment (column 1) or having } \\
\text { had at least one seizure in the } \\
\text { past } 5 \text { years or current } \\
\text { treatment (column 2) }\end{array}$ \\
\hline $\begin{array}{l}\text { Rochester, } \\
\text { MN, U.S.A. } \\
\text { (2) }\end{array}$ & 1991 & $\begin{array}{l}\text { Register based; total } \\
\text { population of Rochester }\end{array}$ & $\begin{array}{c}\mathrm{N}=56,447 \\
\text { (all ages) }\end{array}$ & $\begin{array}{l}55-64 \\
65-74 \\
75+\end{array}$ & $\begin{array}{r}7.7 \\
6.8 \\
14.8\end{array}$ & $\begin{array}{r}9.8 \\
8.1 \\
16.9\end{array}$ & $\begin{array}{l}\text { Recurrent unprovoked seizures; } \\
\text { active defined as having had at } \\
\text { least one seizure in the past } 5 \\
\text { years or having had AED } \\
\text { treatment in the past } 5 \text { years }\end{array}$ \\
\hline $\begin{array}{l}\text { United Kingdom } \\
\text { (3) }\end{array}$ & 1991 & $\begin{array}{l}\text { Register based; } \\
\text { computerized general } \\
\text { practice database }\end{array}$ & $\begin{array}{l}\mathrm{N}=81,727 \\
(60+)\end{array}$ & $\begin{array}{l}60-69 \\
70-79 \\
80+\end{array}$ & $\bar{z}$ & $\begin{array}{l}10.9 \\
12.0 \\
13.1\end{array}$ & $\begin{array}{l}\text { No distinction between provoked } \\
\text { or unprovoked seizures; no } \\
\text { distinction between active } \\
\text { epilepsy or epilepsy in } \\
\text { remission }\end{array}$ \\
\hline Ecuador (18) & 1992 & $\begin{array}{l}\text { Population based; } \\
\text { study-specific screening } \\
\text { protocol }\end{array}$ & $\begin{array}{l}N=10,948 \\
(50+)\end{array}$ & $\begin{array}{l}50-59 \\
60-69 \\
70-98\end{array}$ & $\begin{array}{r}11.7 \\
6.6 \\
8.2\end{array}$ & $\begin{array}{l}21.2 \\
16.4 \\
14.2\end{array}$ & $\begin{array}{l}\text { Single or recurrent seizures, } \\
\text { provoked and unprovoked, } \\
\text { excluding febrile seizures; } \\
\text { active epilepsy defined as } \\
\text { having had a seizure in the past } \\
\text { year and/or receiving AED at } \\
\text { the time of the study }\end{array}$ \\
\hline China (4) & 1985 & $\begin{array}{l}\text { Population based; WHO } \\
\text { screening protocol }\end{array}$ & $\begin{array}{l}N=14,173 \\
(50+)\end{array}$ & $\begin{array}{l}50-59 \\
60-69 \\
70+\end{array}$ & $\overline{-}$ & $\begin{array}{l}3.4 \\
3.9 \\
2.9\end{array}$ & $\begin{array}{l}\text { Recurrent unprovoked seizures; } \\
\text { no distinction between active } \\
\text { and lifetime prevalence }\end{array}$ \\
\hline India (13) & 1988 & $\begin{array}{l}\text { Population based; WHO } \\
\text { screening protocol }\end{array}$ & $\begin{array}{c}N=8,071 \\
(40+)\end{array}$ & $\begin{array}{c}40-59 \\
60+\end{array}$ & $\begin{array}{l}3.4 \\
3.5\end{array}$ & $\begin{array}{l}3.9 \\
4.3\end{array}$ & $\begin{array}{l}\text { Recurrent unprovoked seizures; } \\
\text { active epilepsy defined as } \\
\text { having had at least one seizure } \\
\text { in the past } 5 \text { years regardless of } \\
\text { treatment }\end{array}$ \\
\hline Nigeria (11) & 1987 & $\begin{array}{l}\text { Population based; WHO } \\
\text { screening protocol }\end{array}$ & $\begin{array}{l}\mathrm{N}=18,954 \\
\quad \text { (all ages) }\end{array}$ & $\begin{array}{l}35-54 \\
55+\end{array}$ & $\begin{array}{l}4.5 \\
4.8\end{array}$ & $\overline{-}$ & $\begin{array}{l}\text { Recurrent unprovoked seizures; if } \\
\text { previously untreated, having } \\
\text { had at least one seizure in the } \\
\text { past } 2 \text { years and if under } \\
\text { treatment having had at least } \\
\text { one seizure in the past } 3 \text { years }\end{array}$ \\
\hline Tunesia (12) & 1993 & $\begin{array}{l}\text { Population based; WHO } \\
\text { screening protocol }\end{array}$ & $\begin{array}{c}N=3,460 \\
(50+)\end{array}$ & $\begin{array}{l}50-59 \\
60+\end{array}$ & $\begin{array}{l}0.5 \\
3.1\end{array}$ & $\overline{-}$ & $\begin{array}{l}\text { Recurrent unprovoked seizures; } \\
\text { active defined as having had at } \\
\text { least one seizure in the past } 5 \\
\text { years or having had treatment } \\
\text { in the past } 5 \text { years }\end{array}$ \\
\hline $\begin{array}{l}\text { Copiah } \\
\text { county, } \\
\text { U.S.A. (19) }\end{array}$ & 1986 & $\begin{array}{l}\text { Population based; } \\
\text { study-specific screening } \\
\text { protocol }\end{array}$ & $\begin{array}{c}N=8,925 \\
(40+)\end{array}$ & $\begin{array}{c}40-59 \\
60+\end{array}$ & $\begin{array}{l}8.8 \\
5.1\end{array}$ & $\begin{array}{r}13.1 \\
8.4\end{array}$ & $\begin{array}{l}\text { Recurrent unprovoked seizures; } \\
\text { active defined if under } \\
\text { treatment as having had at least } \\
\text { one seizure in the past } 3 \text { years } \\
\text { and if untreated having had a } \\
\text { seizure in the past year. }\end{array}$ \\
\hline Sweden (5) & 1992 & $\begin{array}{l}\text { Register based; } \\
\text { multisource medical } \\
\text { registers }\end{array}$ & $\begin{array}{c}\mathrm{N}=52,792 \\
(50+)\end{array}$ & $\begin{array}{l}50-59 \\
60-69 \\
70+\end{array}$ & $\begin{array}{l}6.4 \\
6.2 \\
3.2\end{array}$ & $\bar{z}$ & $\begin{array}{l}\text { Recurrent unprovoked seizures; } \\
\text { active defined as having had at } \\
\text { least one seizure in the past } 5 \\
\text { years or receiving AEDs in the } \\
\text { year preceding prevalence date }\end{array}$ \\
\hline Italy (20) & 1983 & $\begin{array}{l}\text { Register based; medical } \\
\text { and nonmedical } \\
\text { registers }\end{array}$ & $\begin{array}{c}\mathrm{N}=45,153 \\
\text { (all ages) }\end{array}$ & $\begin{array}{l}40-59 \\
60+\end{array}$ & $\begin{array}{l}4.6 \\
3.0\end{array}$ & - & $\begin{array}{l}\text { Recurrent unprovoked seizures; } \\
\text { active defined as having had at } \\
\text { least one seizure in the last } 5 \\
\text { years or receiving AEDs }\end{array}$ \\
\hline $\begin{array}{l}\text { United } \\
\text { Kingdom (21) }\end{array}$ & 1983 & $\begin{array}{l}\text { Register based; register of } \\
\text { one general practice }\end{array}$ & $\begin{array}{c}\mathrm{N}=1,581 \\
(51+)\end{array}$ & $\begin{array}{l}50-60 \\
61-70 \\
71-80 \\
80+\end{array}$ & $-^{a}$ & $\begin{array}{l}11.8 \\
24.1 \\
26.2 \\
10.6\end{array}$ & $\begin{array}{l}\text { Provoked and unprovoked } \\
\text { seizures excluding febrile } \\
\text { seizures; active defined as } \\
\text { having had at least one seizure } \\
\text { in the past } 2 \text { years }\end{array}$ \\
\hline
\end{tabular}

AEDs, antiepileptic drugs.

${ }^{a}$ Only an overall prevalence figure for active epilepsy was given: 5.3 in 1,000.

Our study has several possible limitations. First, despite our best efforts, we were unable to obtain $100 \%$ participation. Although our participation rate was high given the age distribution of our study population, the prevalence of epilepsy may be different among nonparticipants as compared with participants. A second issue concerns the sensitivity of our screening questions. The screening instru- 
ment we used contained direct questions regarding epilepsy and current drug use, in addition to questions that were adapted from the WHO protocol originally designed for neuroepidemiological studies in developing countries (7). Other studies have also used this protocol to screen for epilepsy $(4,11-$ 13). Nonetheless, although high sensitivities have been reported, the instrument has never been validated properly (14). We did not include a sample of screen negatives because the prevalence among screen negatives is likely to be much lower than that in the total study population, and to obtain a stable and precise estimate of the prevalence among screen negatives, a very large sample would have been required. Therefore, we may have missed some cases of epilepsy, especially persons with partial seizures. Finally, Alzheimer's disease is a recognized risk factor for late-onset epilepsy $(15,16)$. Because of underreporting of seizures and symptoms in demented patients, we may have missed some patients with both dementia and epilepsy. These various considerations make it likely that our prevalence figures are slight underestimates.

Comparisons among studies must take into account variations in methods and definitions (2). Table 7 compares the results of our study with those of several other recent investigations which provided age-specific prevalences of epilepsy. We classified special syndromes as epilepsy according to the 1989 ILAE classification (9). The rationale of including special syndrome is debatable, however. Most acute symptomatic seizures are clearly not epilepsy, and some subjects who immediately receive treatment with AEDs after a first cryptogenic or remote symptomatic seizure may or may not develop epilepsy subsequently. Furthermore, single seizures that occurred long ago are likely to be underreported. To enhance comparability, we report rates for active and lifetime epilepsy that exclude the special syndromes.

Few investigators have reported age-specific prevalence figures for active epilepsy in the elderly, and those that did generally provided rates considerably lower than those we report. Screening for epilepsy relies on a willingness to report epilepsy or seizures or related symptoms. In the Rotterdam Study, screening for epilepsy was conducted as part of a much larger investigation of other diseases relevant to aging and the participation rate was high (6). This study context may have contributed to our identifying a large number of patients. For lifetime prevalence, however, our estimates are relatively low, in particular as compared with estimates from register-based studies. Because remission rates are estimated to exceed $61 \%$ at 10 years after of epi- lepsy onset (17), population-based studies of the lifetime prevalence of epilepsy may well suffer from recall bias and underreporting, resulting in considerable underestimation, especially in the elderly. Besides, what researchers truly wish to ascertain is the cumulative incidence. Because mortality is increased among subjects with epilepsy, as compared to subjects without epilepsy, lifetime prevalence will always underestimate cumulative incidence.

The results of our study confirm earlier findings indicating that the prevalence of active epilepsy increases with age in the elderly. The prevalence figures in our study were high as compared with those of other population-based studied. Epilepsy appears to be a major cause of morbidity in the elderly.

Acknowledgment: This work was supported in part by Project No. CLEO A-106 from the Committee on Epilepsy of the Division for Health Research of the National Epilepsy Fund 'The Power of the Tiny', the NESTOR program for geriatric research (Ministry of Health and Ministry of Education), The Netherlands Organisation for scientific research (NWO), and the Municipality of Rotterdam. We thank Drs. D. J. Beintema, C. A. E. H. van Oorschot, A. van Wieringen, and J. M. de WildeOckelone for diagnostic contributions and Dr. M. J. Rutgers and the late Professor P. van der Lugt for helping plan the study.

\section{REFERENCES}

1. Hauser WA, Kurland LT. The epidemiology of epilepsy in Rochester, Minnesota, 1935 through 1967. Epilepsia 1975; 16:1-66.

2. Hauser WA, Annegers JF, Kurland LT. Prevalence of epilepsy in Rochester, Minnesota, 1940-1980. Epilepsia 1991; $31: 429-45$.

3. Tallis R, Hall G, Craig I, Dean A. How common are epileptic seizures in old age? Age Ageing 1991;20:442-8.

4. Li S, Schoenberg BS, Wang C, Cheng X, Zhou S, Bolis CL. Epidemiology of epilepsy in urban areas of the People's Republic of China. Epilepsia 1985;26:391-4.

5. Forsgren L. Prevalence of epilepsy in adults in Northern Sweden. Epilepsia 1992;33:450-8.

6. Hofman A, Grobbee DE, De Jong PTVM, Van den Ouweland FA. Determinants of disease and disability in the elderly: the Rotterdam Elderly Study. Eur J Epidemiol 1991;7: 403-22.

7. World Health Organization. Research protocol for measuring the prevalence of neurological disorders in developing countries. Geneva: World Health Organization, Neurosciences Program, 1981.

8. Anatomical Therapeutic Chemical (ATC) classification index. Oslo: WHO Collaborating Centre for Drug Statistics Methodology, 1992.

9. Commission on Classification and Terminology of the International League Against Epilepsy: proposal for revised classification of epilepsies and epileptic syndromes. Epilepsia 1989;30:389-99.

10. Commission on epilepsy and prognosis, International League Against Epilepsy. Guidelines for epidemiologic studies on epilepsy. Epilepsia 1993;34:592-6.

11. Osuntokun BO, Adeuja AOG, Nottidge VA, et al. Preva- 
lence of the epilepsies in Nigerian Africans: a communitybased study. Epilepsia 1987;28:272-9.

12. Attia-Romdhane N, Mrabet A, Ben Hamida M. Prevalence of epilepsy in Kelibia, Tunesia. Epilepsia 1993;34:1028-32.

13. Bharucha NE, Bharucha EP, Bharucha AE, Bhise AV, Schoenberg BS. Prevalence of epilepsy in the Parsi Community of Bombay. Epilepsia 1988;29:111-5.

14. Placencia JW, Sander JWAS, Shorvon SD, Ellison RH, Cascante SM. Validation of a screening questionnaire for the detection of epileptic seizures in epidemiological studies. Brain 1992;115:783-94.

15. Romanelli MF, Morris JC, Ashkin KA, Cohen LA. Advanced Alzheimer's disease is a risk factor for late-onset seizures. Arch Neurol 1990;47:847-50.

16. Hauser WA, Morris ML, Heston LL, Anderson VE. Sei- zures and myoclonus in patients with Alzheimer's disease Neurology 1986;36:1226-30.

17. Annegers JF, Hauser WA, Elverback LR. Remission of seizures and relapse in patients with epilepsy. Epilepsia 1979; 20:729-37.

18. Placencia M, Shorvon SD, Paredes V, et al. Epileptic seizures in an Andean region of Ecuador. Brain 1992;115:77182.

19. Haerer AF, Anderson DW, Schoenberg BS. Prevalence and clinical features of epilepsy in a biracial United States population. Epilepsia 1986;27:66-75.

20. Granieri E, Rosati G, Tola R, et al. A descriptive study of epilepsy in the district of Copparo, Italy, 1964-1978. Epilepsia $1983 ; 24: 502-14$.

21. Goodridge DMG, Shorvon SD. Epileptic seizures in a population of 6000. Br Med J 1983;287:641-7. 